\title{
Focus on computational and systems neuroscience
}

\author{
We present a special focus on computational and systems neuroscience, highlighting recent advances in combining \\ empirical and theoretical approaches, including work presented at the Cosyne meeting in past years.
}

T his month Nature Neuroscience brings you a special focus on computational and systems neuroscience. Some long-time readers may have a sense of déjà vu-haven't we done this before? In fact, we have-just about five years ago. So, why do it again? In 2005, the goal of the focus was to bring attention to a newly burgeoning field and to encourage empiricists and theoreticians to work together to generate novel syntheses. Over the past five years, the field has grown considerably: the number of computational neuroscience papers listed in PubMed has nearly doubled. The current focus is a celebration of a field that has come into its own. In this month's issue, we present perspective and review articles that we hope will give our readers a broad sense of the some of the more recent advances in this field, as well as basic research articles from work presented at one of the main meetings in this area: the Computational and Systems Neuroscience meeting, Cosyne (http://www.cosyne.org/).

One of the biggest technical changes in the field of computational and systems neuroscience has been the dramatic increase in the number of neurons that can be simultaneously recorded. Reports of single-cell and tetrode recordings were once the norm, with the occasional data set from a hand-made electrode array. Prefabricated arrays can now be easily purchased and multichannel recordings are commonplace, enabling researchers to ask questions about the activity of entire networks of neurons. On page 139, Stevenson and Kording highlight the scale of this escalation: the number of simultaneously recorded neurons has doubled approximately every seven years. Consequently, they suggest that emerging data analysis techniques should consider both the computational costs and the potential for more accurate models that exploit this exponential growth in the size of the neural network that can be recorded.

Simultaneous recordings from multiple neurons has also been a big benefit to those who are interested the interactions between neurons at the circuit level. However, the diversity of properties that can be measured even among the same identified cells in a circuit such as the stomatogastric ganglion has generally been thought of as a bug, rather than a feature of neural circuitry. Eve Marder and her colleagues have long espoused the view that this variability is not simply noise, but information about how cells and circuits might develop compensatory mechanisms to produce the same function. On page 133, Marder and Taylor suggest that in using theoretical tools to investigate this possibility, a profitable approach would be to construct a population of models with different underlying structures that give rise to similar behaviors.

Multiple-neuron recordings are not novel to those studying replay activity in the hippocampus either. Although these studies are not always thought about in the context of computational and systems neuroscience, sophisticated computational techniques have been critical for extracting reactivated sequences of activity from the many ongoing signals recorded across the hippocampus, and theoretical frameworks have been helpful in understanding the links between these neural signals and behavior. Replay was first identified in sleeping rats, suggesting that it subserves memory consolidation. More recently, replay activity has been identified in awake animals as well. On page 147, Loren Frank and colleagues review these recent findings and discuss a potential function of awake replay in learning and memory consolidation.

Another area where theoretical frameworks have been helpful in understanding behavior has been decision making in animals. In a perspective on page 143, David Freedman and John Assad discuss the role of lateral intraparietal cortex (LIP) in decision making. Empiricists have been trying to discern the function of area LIP for a long time, and several computational frameworks have been brought to bear on the question. Although much ink has been spilled on this subject, these lines of evidence have not yet quite converged. Here Freedman and Assad propose a synthesis of the previous work that suggests a more unified view.

Computational models of behavior can also help researchers and clinicians make sense of the complex empirical observations of human patients in the psychiatric clinic. On page 154, Michael Frank and Tiago Maia review the burgeoning field of computational psychiatry. They describe how the now ubiquitous reinforcement learning models can be applied to bring new insight into disorders from Parkinson's disease to schizophrenia and beyond.

As in our previous focus on this field, we are taking the unusual step of including primary research papers besides the commissioned reviews and perspectives. These research papers include some of the best work previously presented at the Cosyne meeting. This meeting has been held every year since 2004 and brings together a broad range of theorists and experimentalists interested in systems neuroscience. Reflecting the diversity of attendees at the meeting, the papers span a variety of topics and contain different degrees of theoretical formalization. Every research article in this special issue was subjected to our regular peerreview process. We applied our usual stringent editorial standards to each paper, and each one met the criteria for publication in a regular issue of Nature Neuroscience.

Of course, these reviews, perspectives and research articles cannot possibly cover everything that could be considered computational and systems neuroscience. However, we hope that the work presented in this special issue, assembled by Senior Editor Hannah Bayer, provide a tantalizing sample of the progress being made in the field in computational and systems neuroscience. We are very grateful to our authors, reviewers and advisors for their help with this collection. 